\title{
Classification of families of pr- and epr-sequences
}

\author{
Xavier Martínez-Rivera* \\ Department of Mathematics, Iowa State University, Ames, IA, USA
}

September 5, 2018

\begin{abstract}
This paper establishes new restrictions for attainable enhanced principal rank characteristic sequences (epr-sequences). These results are then used to classify two related families of sequences that are attainable by a real symmetric matrix: the family of principal rank characteristic sequences (pr-sequences) not containing three consecutive 1s and the family of epr-sequences which contain an $\mathrm{N}$ in every subsequence of length 3 .
\end{abstract}

Keywords: Principal rank characteristic sequence; enhanced principal rank characteristic sequence; minor; rank; symmetric matrix

AMS Subject Classifications: 15A15; 15A03; 15B57

\section{Introduction}

Given an $n \times n$ symmetric matrix $B$ over a field $F$, the principal rank characteristic sequence (abbreviated pr-sequence) of $B$ is defined as $\left.\operatorname{pr}(B)=r_{0}\right] r_{1} \cdots r_{n}$, where

$$
r_{k}= \begin{cases}1 & \text { if } B \text { has a nonzero principal minor of order } k, \text { and } \\ 0 & \text { otherwise }\end{cases}
$$

while $r_{0}=1$ if and only if $B$ has a 0 diagonal entry [2]; the order of a minor is $k$ if it is the determinant of a $k \times k$ submatrix.

The principal minor assignment problem, introduced in [5], asks the following question: can we find an $n \times n$ matrix with prescribed principal minors? As a simplification of the principal minor assignment problem, Brualdi et al. [2] introduced the pr-sequence of a real symmetric matrix as defined above. An attractive result obtained in [2] is the requirement

${ }^{*}$ Corresponding author. Email: xaviermr@iastate.edu 
that a pr-sequence that can be realized by a real symmetric matrix cannot contain the subsequence 001, meaning that in the pr-sequence of such matrix, the presence of the subsequence 00 forces 0 s from that point forward. This result was later generalized by Barrett et al. [1] for symmetric matrices over any field; this led them to the study of symmetric matrices over various fields, where, among other results, a characterization of the pr-sequences that can be realized by a symmetric matrix over a field of characteristic 2 was obtained. Although not deeply studied, the family of pr-sequences not containing three consecutive 1 s were of interest in [2], since the pr-sequences of the principal submatrices of a matrix realizing a pr-sequence not containing three consecutive 1s possess the rare property of being able to inherit the majority of the 1s of the original sequence; this family will be one of the central themes of this paper.

Due to the limitations of the pr-sequence, which only records the presence or absence of a full-rank principal submatrix of each possible order, Butler et al. 3 introduced the the enhanced principal rank characteristic sequence (abbreviated epr-sequence) of an $n \times n$ symmetric matrix $B$ over a field $F$, denoted by $\operatorname{epr}(B)=\ell_{1} \ell_{2} \cdots \ell_{n}$, where

$$
\ell_{k}= \begin{cases}\mathrm{A} & \text { if all the principal minors of order } k \text { are nonzero; } \\ \mathrm{S} & \text { if some but not all the principal minors of order } k \text { are nonzero; } \\ \mathrm{N} & \text { if none of the principal minors of order } k \text { are nonzero, i.e., all are zero. }\end{cases}
$$

A (pr- or epr-) sequence is said to be attainable over a field $F$ provided that there exists a symmetric matrix $B \in F^{n \times n}$ that attains it; otherwise, we say that it is unattainable. Among other results, techniques to construct attainable epr-sequences were presented in [3], as well as necessary conditions for an epr-sequence to be attainable by a symmetric matrix, with many of them asserting that subsequences such as NSA, NAN and NAS, among others, cannot occur in epr-sequences over certain fields. Continuing the study of epr-sequences, Fallat et al. 4] characterized all the epr-sequences that are attainable by skew-symmetric matrices.

In this paper the study of pr- and epr-sequences of symmetric matrices is continued. Section 2 establishes new restrictions for epr-sequences to be attainable over certain fields. The results from Section 2 are then implemented in Section 3. where, for real symmetric matrices, we classify all the attainable pr-sequences not containing three consecutive 1s. Using this classification, in Section 4, a related family of attainable epr-sequences is classified, namely those that contain an $\mathrm{N}$ in every subsequence of length 3 . We then conclude with Proposition 4.6, where we highlight an interesting property exhibited by the vast majority of attainable pr-sequences not containing three consecutive 1s; that is, the property of being associated with a unique attainable epr-sequence.

A pr-sequence and an epr-sequence are associated with each other if a matrix (which may not exist) attaining the epr-sequence also attains the pr-sequence. A subsequence that does not appear in an attainable sequence is forbidden (and we may also say that it is prohibited). Moreover, a sequence is said to have order $n$ if it corresponds to a matrix of order $n$, while a subsequence has length $n$ if it consists of $n$ terms.

Let $B=\left[b_{i j}\right]$ and let $\alpha, \beta \subseteq\{1,2, \ldots, n\}$. Then the submatrix lying in rows indexed by $\alpha$, and columns indexed by $\beta$, is denoted by $B[\alpha, \beta]$; if $\alpha=\beta$, then $B[\alpha, \alpha]$ is abbreviated to $B[\alpha]$. The matrices $0_{n}, I_{n}$ and $J_{n}$ are the matrices of order $n$ denoting the zero matrix, the identity matrix and the all-1s matrix, respectively. The direct sum of two matrices $B$ and 
$C$ is denoted by $B \oplus C$. Given a graph $G, A(G)$ denotes the adjacency matrix of $G$, while $P_{n}$ and $C_{n}$ denote the path and cycle, respectively, on $n$ vertices.

\section{$1.1 \quad$ Results cited}

The purpose of this section is to list results we will cite frequently, and assign abbreviated nomenclature to some of them.

Theorem 1.1. 2, Theorem 2.7] Suppose $B$ is a nonsingular real symmetric matrix with $\left.\operatorname{pr}(B)=r_{0}\right] r_{1} \cdots r_{n}$. Let $\left.\operatorname{pr}\left(B^{-1}\right)=r_{0}^{\prime}\right] r_{1}^{\prime} \cdots r_{n}^{\prime}$. Then $r_{n}^{\prime}=r_{n}=1$, while for each $i$ with $1 \leq i \leq n-1, r_{i}^{\prime}=r_{n-i}$. Finally, $r_{0}^{\prime}=1$ if and only if $B$ has some principal minor of order $n-1$ that is zero.

Theorem 1.2. [2, Theorem 4.4] (00 Theorem) Let $B$ be a real symmetric matrix. Let $\left.\operatorname{pr}(B)=r_{0}\right] r_{1} \cdots r_{n}$ and suppose that, for some $k$ with $0 \leq k \leq n-2, r_{k+1}=r_{k+2}=0$. Then $r_{i}=0$ for all $i \geq k+1$. In particular, $r_{n}=0$, so that $B$ is singular.

Theorem 1.3. [2, Theorem 6.5] (0110 Theorem) Suppose $n \geq 4$ and $\left.p r(B)=r_{0}\right] r_{1} \cdots r_{n}$. If, for some $k$ with $1 \leq k \leq n-3, r_{k}=r_{k+3}=0$, then $r_{i}=0$ for all $k+3 \leq i \leq n$. In particular, $B$ is singular.

A generalization of Theorem 1.2 in [1] led to an analogous result for epr-sequences over any field:

Theorem 1.4. [3, Theorem 2.3] (NN Theorem) Suppose $B$ is a symmetric matrix over a field $F, \operatorname{epr}(B)=\ell_{1} \ell_{2} \cdots \ell_{n}$, and $\ell_{k}=\ell_{k+1}=\mathrm{N}$ for some $k$. Then $\ell_{i}=\mathrm{N}$ for all $i \geq k$. (That $i s$, if an epr-sequence of a matrix ever has $\mathrm{NN}$, then it must have $\mathrm{N} s$ from that point forward.)

Theorem 1.5. [3, Theorem 2.4] (Inverse Theorem) Suppose $B$ is a nonsingular symmetric matrix over a field $F$. If $\operatorname{epr}(B)=\ell_{1} \ell_{2} \cdots \ell_{n-1} \mathrm{~A}$, then $\operatorname{epr}\left(B^{-1}\right)=\ell_{n-1} \ell_{n-2} \cdots \ell_{1} \mathrm{~A}$.

Each instance of $\cdots$ below is permitted to be empty.

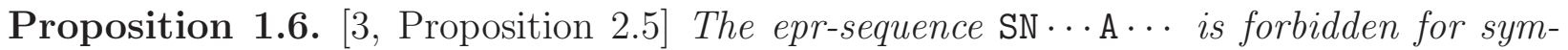
metric matrices over any field.

We say that SN $\cdots A$ A $\cdots$ is prohibited when referencing Proposition 1.6.

Theorem 1.7. [3, Theorem 2.6] (Inheritance Theorem) Suppose that $B$ is a symmetric matrix over a field $F, m \leq n$, and $1 \leq i \leq m$.

1. If $[\operatorname{epr}(B)]_{i}=\mathrm{N}$, then $[\operatorname{epr}(C)]_{i}=\mathrm{N}$ for all $m \times m$ principal submatrices $C$.

2. If $[\operatorname{epr}(B)]_{i}=\mathrm{A}$, then $[\operatorname{epr}(C)]_{i}=\mathrm{A}$ for all $m \times m$ principal submatrices $C$.

3. If $[\operatorname{epr}(B)]_{m}=\mathrm{S}$, then there exist $m \times m$ principal submatrices $C_{A}$ and $C_{N}$ of $B$ such that $\left[\operatorname{epr}\left(C_{A}\right)\right]_{m}=\mathrm{A}$ and $\left[\operatorname{epr}\left(C_{N}\right)\right]_{m}=\mathrm{N}$.

4. If $i<m$ and $[\operatorname{epr}(B)]_{i}=\mathrm{S}$, then there exists an $m \times m$ principal submatrix $C_{S}$ such that $\left[\operatorname{epr}\left(C_{S}\right)\right]_{i}=\mathrm{S}$. 
Corollary 1.8. [3, Corollary 2.7] No symmetric matrix over any field can have NSA in its epr-sequence. Further, no symmetric matrix over any field can have the epr-sequence $\cdots$ ASN $\cdots$ A $\cdots$.

Corollary 1.8 will be invoked by just stating that NSA or $\cdots$ ASN $\cdots A$ A is prohibited.

If $B$ is a matrix with a nonsingular principal submatrix $B[\alpha], B / B[\alpha]$ denotes the Schur complement of $B[\alpha]$ in $B$.

Theorem 1.9. [3, Proposition 2.13] (Schur Complement Theorem) Suppose B is a symmetric matrix over a field of characteristic not 2 with $\operatorname{rank} B=m$. Let $B[\alpha]$ be a nonsingular principal submatrix of $B$ with $|\alpha|=k \leq m$, and let $C=B / B[\alpha]$. Then the following results hold.

1. $C$ is an $(n-k) \times(n-k)$ symmetric matrix.

2. Assuming the indexing of $C$ is inherited from $B$, any principal minor of $C$ is given by $\operatorname{det} C[\gamma]=\operatorname{det} B[\gamma \cup \alpha] / \operatorname{det} B[\alpha]$.

3. $\operatorname{rank} C=m-k$.

4. Any nonsingular principal submatrix of $B$ of order at most $m$ is contained in a nonsingular principal submatrix of order $m$.

Theorem 1.10. [3, Theorem 2.14] Neither the epr-sequences NAN nor NAS can occur as a subsequence of the epr-sequence of a symmetric matrix over a field of characteristic not 2.

We will refer to Theorem 1.10 by simply stating that NAN or NAS is prohibited, while Theorem 1.11 below is referenced by stating that ANS 'must be initial.'

Theorem 1.11. [3, Theorem 2.15] In the epr-sequence of a symmetric matrix over a field of characteristic not 2 , the subsequence ANS can only occur as the initial subsequence.

\section{Restrictions on attainable epr-sequences}

In this section, we establish new restrictions on attainable epr-sequences. We begin with restrictions that apply to fields of characteristic not 2. For convenience, given a matrix $B$, we adopt some of the notation in [2], and denote with $B_{i_{1} i_{2} \ldots i_{k}}$, the principal minor $\operatorname{det}\left(B\left[\left\{i_{1}, i_{2}, \ldots, i_{k}\right\}\right]\right)$.

Proposition 2.1. Let $n \geq 6$. Then no $n \times n$ symmetric matrix over a field of characteristic not 2 has an epr-sequence starting NSNA $\cdots$.

Proof. Let $B=\left[b_{i j}\right]$ be an $n \times n$ symmetric matrix over a field of characteristic not 2 and let $\operatorname{epr}(B)=\ell_{1} \ell_{2} \cdots \ell_{n}$. Suppose to the contrary that $\operatorname{epr}(B)=$ NSNA $\cdots$. Since $\ell_{3}=N$, and because $B_{p q r}=2 b_{p q} b_{p r} b_{q r}$ for any distinct $p, q, r \in\{1,2, \ldots, n\}, B[\{1,2,3\}]$ and $B[\{4,5,6\}]$ must each contain a zero off-diagonal entry. Moreover, since $\ell_{4}=\mathrm{A}, 0_{3}$ is not a principal submatrix of $B$, implying that $B[\{1,2,3\}]$ and $B[\{4,5,6\}]$ must each contain a nonzero 
off-diagonal entry. Since $\{1,2,3\}$ and $\{4,5,6\}$ are disjoint, and because a simultaneous permutation of the rows and columns of a matrix has no effect on its determinant, we may assume, without loss of generality, that $b_{12}=b_{56}=0$ and that $b_{13}, b_{46}$ are nonzero. Similarly, since $\{1,2,3\}$ and $\{4,5,6\}$ are disjoint, and because multiplication of any row and column of a matrix by a nonzero constant preserves the rank of every submatrix, we may also assume, without loss of generality, that $b_{13}=b_{46}=1$. We consider two cases.

Case 1: $b_{14}=0$. Since $\ell_{4}=\mathrm{A},\left(b_{15} b_{24}\right)^{2}=B_{1245} \neq 0$; it follows that $b_{15}$ and $b_{24}$ are nonzero. Since $\ell_{3}=N, B_{135}=2 b_{15} b_{35}=0$; hence, $b_{35}=0$. Since $B[\{3,5,6\}] \neq 0_{3}, b_{36} \neq 0$. Since $2 b_{16} b_{36}=B_{136}=0, b_{16}=0$. Then, as $B[\{1,2,6\}] \neq 0_{3}, b_{26} \neq 0$. It follows that $B_{246}=2 b_{24} b_{26} \neq 0$, a contradiction to $\ell_{3}=\mathrm{N}$, implying that it is impossible to have $b_{14}=0$.

Case 2: $b_{14} \neq 0$. Since $2 b_{14} b_{34}=B_{134}=0$, and because $2 b_{14} b_{16}=B_{146}=0, b_{34}=b_{16}=0$. Since $B[\{1,2,6\}] \neq 0_{3}, b_{26} \neq 0$. Since $2 b_{24} b_{26}=B_{246}=0, b_{24}=0$. Since $\left(b_{14} b_{23}\right)^{2}=B_{1234} \neq$ $0, b_{23} \neq 0$. Then, as $2 b_{23} b_{26} b_{36}=B_{236}=0, b_{36}=0$. It follows that $B_{1356}=0$, a contradiction to $\ell_{4}=\mathrm{A}$.

It should be noted that NSNA and NSNAA are attainable by $A\left(P_{4}\right)$ and $A\left(C_{5}\right)$, respectively [3], but this does not contradict Proposition [2.1, which requires $n \geq 6$.

Proposition 2.2. Let $B$ be a symmetric matrix over a field of characteristic not 2 and $\operatorname{epr}(B)=\ell_{1} \ell_{2} \cdots \ell_{n}$. Then NSNA cannot occur as a subsequence of $\ell_{1} \ell_{2} \cdots \ell_{n-2}$.

Proof. If $n \leq 5$, the result follows vacuously. So, assume $n \geq 6$. Suppose to the contrary that NSNA occurs as a subsequence of $\ell_{1} \ell_{2} \cdots \ell_{n-2}$ and that $\ell_{k} \ell_{k+1} \ell_{k+2} \ell_{k+3}=$ NSNA, for some $k$ with $1 \leq k \leq n-5$. By Proposition 2.1 , $k \geq 2$, and, by the NN Theorem, $\ell_{k-1} \neq \mathrm{N}$; it follows that $B$ has a $(k-1) \times(k-1)$ nonsingular principal submatrix, say $B[\alpha]$. By the Schur Complement Theorem, $B / B[\alpha]$ has an epr-sequence starting NXNAYZ $\cdots$, where $\mathrm{X}, \mathrm{Y}, \mathrm{Z} \in\{\mathrm{A}, \mathrm{S}, \mathrm{N}\}$. The NN Theorem and the fact that NAN is prohibited imply that $\mathrm{X}=\mathrm{S}$; hence, $\operatorname{epr}(B)$ starts NSNAYZ $\cdots$, a contradiction to Proposition 2.1.

With the next result, we generalize (and provide a simpler proof of) [3, Proposition 2.11].

Proposition 2.3. Suppose $B$ is a symmetric matrix over a field of characteristic not 2, $\operatorname{epr}(B)=\ell_{1} \ell_{2} \cdots \ell_{n}$ and $\ell_{k} \ell_{k+1} \ell_{k+2}=$ SAN for some $k$. Then $\ell_{j}=\mathrm{N}$ for all $j \geq k+2$.

Proof. If $n=3$, we are done. Suppose $n>3$. Suppose that $\ell_{k} \ell_{k+1} \ell_{k+2}=$ SAN for some $k$ with $1 \leq k \leq n-2$. If $k=n-2$, we are done. Suppose $k<n-2$. By [3. Corollary 2.10], which prohibits SANA, $\ell_{k+3} \neq \mathrm{A}$. Since ANS must be initial, $\ell_{k+3} \neq \mathrm{S}$. Hence, $\ell_{k+3}=\mathrm{N}$. The desired conclusion now follows from the NN Theorem.

We now confine our attention to real symmetric matrices. The next result is immediate from Theorem [1.3.

Proposition 2.4. Let $B$ be a real symmetric matrix and $\operatorname{epr}(B)=\ell_{1} \ell_{2} \cdots \ell_{n}$. Suppose $\ell_{k}=\ell_{k+3}=\mathrm{N}$ for some $k \geq 1$. Then $\ell_{i}=\mathrm{N}$ for all $i \geq k+3$. In particular, $B$ is singular.

We emphasize that Proposition 2.4 asserts that a sequence of the form $\cdots$ NXYN $\cdots Z \cdots$, with $\mathrm{X}, \mathrm{Y} \in\{\mathrm{A}, \mathrm{S}, \mathrm{N}\}$ and $\mathrm{Z} \in\{\mathrm{A}, \mathrm{S}\}$, is not attainable by a real symmetric matrix.

Given a sequence $t_{i_{1}} t_{i_{2}} \cdots t_{i_{k}}, \overline{t_{i_{1}} t_{i_{2}} \cdots t_{i_{k}}}$ indicates that the sequence may be repeated as many times as desired (or it may be omitted entirely). According to [․․, Proposition 2.17], 
the sequence ANA $\bar{A}$ is attainable by a symmetric matrix over a field of characteristic 0 . [3, Table 1] raises the following question: does a real symmetric matrix, with an epr-sequence starting ANA $\cdots$, always have epr-sequence ANA $\bar{A}$ ? The answer is affirmative; what follows makes this precise.

Proposition 2.5. Any $n \times n$ real symmetric matrix with an epr-sequence starting ANA... is conjugate by a nonsingular diagonal matrix to one of $\pm\left(J_{n}-2 I_{n}\right)$. Furthermore, its eprsequence is ANA $\bar{A}$.

Proof. Let $B=\left[b_{i j}\right]$ be an $n \times n$ real symmetric matrix with an epr-sequence starting ANA $\cdots$. Notice that all the diagonal entries of $B$ must have the same sign, as otherwise there would be a principal minor of order 2 that is nonzero. Let $C=\left[c_{i j}\right]$ be the matrix among $B$ and $-B$ with all diagonal entries negative. Let $D=\left[d_{i j}\right]$ be the $n \times n$ diagonal matrix with $d_{11}=1 / \sqrt{-c_{11}}$ and $d_{j j}=\operatorname{sign}\left(c_{1 \mathrm{j}}\right) / \sqrt{-\mathrm{c}_{\mathrm{jj}}}$ for $j \geq 2$. Now, notice that every entry of $D C D$ is \pm 1 , every diagonal entry is -1 and every off-diagonal entry in the first row and the first column is 1 . We now show that $D C D=J_{n}-2 I_{n}$. Since multiplication of any row and column of a matrix by a nonzero constant preserves the rank of every submatrix, $\operatorname{epr}(D C D)=\operatorname{epr}(C)=\operatorname{epr}(B)$. Let $i, j \in\{2,3, \ldots n\}$ be distinct, $\alpha=\{1, i, j\}$ and let $a$ be the $(i, j)$-entry of $D C D$. A simple computation shows that $\operatorname{det}((D C D)[\alpha])=(a+1)^{2}$. Since every principal minor of order 3 of $D C D$ is nonzero, $a=1$. Then, as $i$ and $j$ were arbitrary, $D C D=J_{n}-2 I_{n}$. Then, as $C=B$ or $C=-B$, it follows that $B$ is conjugate by a nonsingular diagonal matrix to one of $\pm\left(J_{n}-2 I_{n}\right)$, and that $\operatorname{epr}(B)=\operatorname{epr}\left(J_{n}-2 I_{n}\right)=\operatorname{ANA} \overline{\mathrm{A}}$ (see [3, Proposition 2.17]).

We are now in position to prove the following result.

Theorem 2.6. Any epr-sequence of a real symmetric matrix containing ANA as a nonterminal subsequence is of the form $\overline{\mathrm{A} A N A} \mathrm{~A} \overline{\mathrm{A}}$.

Proof. Let $B$ be a real symmetric matrix containing ANA as a non-terminal subsequence. Let $\operatorname{epr}(B)=\ell_{1} \ell_{2} \cdots \ell_{n}$. Suppose $\ell_{k+1} \ell_{k+2} \ell_{k+3}=$ ANA for some $k$ with $0 \leq k \leq n-4$. Since NAN and NAS are prohibited, $\ell_{k+4}=\mathrm{A}$. If $k=0$, the conclusion follows from Proposition 2.5, so, assume $k>0$. Suppose $\ell_{i} \neq \mathrm{A}$ for some $i$ with $i<k+1$. By the Inheritance Theorem, $B$ has a (nonsingular) $(k+4) \times(k+4)$ principal submatrix $B^{\prime}$ whose epr-sequence $\ell_{1}^{\prime} \ell_{2}^{\prime} \cdots \ell_{k+4}^{\prime}$ ends with ANAA and has $\ell_{i}^{\prime} \neq \mathrm{A}$. Then, by the Inverse Theorem, $\operatorname{epr}\left(\left(B^{\prime}\right)^{-1}\right)$ starts with ANA and $\operatorname{epr}\left(\left(B^{\prime}\right)^{-1}\right) \neq$ ANAA, a contradiction to Proposition 2.5. Thus, $\operatorname{epr}(B)=\overline{\mathrm{A} A A N A A} \ell_{k+5} \cdots \ell_{n}$, where $\ell_{k+5} \cdots \ell_{n}$ may not exist.

We now show that $\ell_{k+5} \cdots \ell_{n}=\overline{\mathrm{A}}$. If $n=k+4$, we are done; so, suppose $n>k+4$. We proceed by contradiction, and consider two cases.

Case $1: \ell_{j}=\mathrm{N}$ for some $j>k+4$. Since $\ell_{k}=\mathrm{A}$, there exists a $k \times k$ principal submatrix of $B$, say $B[\alpha]$, that is nonsingular. Let $C=B / B[\alpha]$. By the Schur Complement Theorem, $C$ has order $n-k, \operatorname{epr}(C)$ starts ANA $\cdots$ and $\operatorname{epr}(C)$ has an $\mathrm{N}$ in the $(j-k)$-th position; hence, $\operatorname{epr}(C) \neq$ ANA $\bar{A}$, a contradiction to Proposition 2.5. It follows that a sequence containing ANA as a non-terminal subsequence cannot contain an $\mathrm{N}$ from that point forward, implying that any real symmetric matrix with an epr-sequence containing ANA is nonsingular.

Case 2: $\ell_{j}=\mathrm{S}$ for some $j>k+4$. By the Inheritance Theorem, $B$ has a singular $j \times j$ principal submatrix whose epr-sequence contains ANA, which contradicts the assertion above. 
We conclude that we must have $\ell_{k+5} \cdots \ell_{n}=\overline{\mathrm{A}}$, which completes the proof.

It is natural to now ask, does Theorem 2.6 hold if ANA occurs at the end of the sequence? According to [3, Table 5], SAANA is attainable, answering the question negatively.

Theorem 2.7. Let $B$ be a real symmetric matrix with $\operatorname{epr}(B)=\ell_{1} \ell_{2} \cdots \ell_{n}$. Then SNA cannot occur as a subsequence of $\ell_{1} \ell_{2} \cdots \ell_{n-2}$.

Proof. If $n \leq 4$, the result follows vacuously. So, assume $n>4$. Suppose to the contrary that SNA occurs as a subsequence of $\ell_{1} \ell_{2} \cdots \ell_{n-2}$, and that $\ell_{k+1} \ell_{k+2} \ell_{k+3}=$ SNA for some $k$ with $0 \leq k \leq n-5$. Since SN $\cdots A \cdot \cdots$ is prohibited, $k \geq 1$. Since NAN and NAS are prohibited, $\ell_{k+4}=\mathrm{A}$. Then, as ASNA is prohibited, $\ell_{k} \neq \mathrm{A}$. And, by Proposition $2.2, \ell_{k} \neq \mathrm{N}$; it follows that $\ell_{k}=\mathrm{S}$. Thus, we have $\ell_{k} \cdots \ell_{k+4}=$ SSNAA. We examine the three possibilities for $\ell_{k+5}$.

Case 1: $\ell_{k+5}=\mathrm{A}$. Now we have $\ell_{k} \cdots \ell_{k+5}=$ SSNAAA. By the Inheritance Theorem, $B$ has a $(k+5) \times(k+5)$ principal submatrix $B^{\prime}$ whose epr-sequence ends with SXNAAA, where $\mathrm{X} \in\{\mathrm{A}, \mathrm{S}, \mathrm{N}\}$. By the NN Theorem, $\mathrm{X} \neq \mathrm{N}$; and, by Proposition $2.3, \mathrm{X} \neq \mathrm{A}$; it follows that $\mathrm{X}=\mathrm{S}$. By the Inverse Theorem, $\operatorname{epr}\left(\left(B^{\prime}\right)^{-1}\right)$ contains ANS as a non-initial subsequence, a contradiction, since ANS must be initial. We conclude that $\ell_{k+5} \neq \mathrm{A}$.

Case 2: $\ell_{k+5}=$ N. Now we have $\ell_{k} \cdots \ell_{k+5}=$ SSNAAN. Since $\ell_{k}=\mathrm{S}, B$ has a $k \times k$ nonsingular principal submatrix, say $B[\alpha]$. By the Schur Complement Theorem, $B / B[\alpha]$ has an epr-sequence starting YNAAN $\cdots$, where $\mathrm{Y} \in\{\mathrm{A}, \mathrm{S}, \mathrm{N}\}$. By Theorem 2.6, $\mathrm{Y} \neq \mathrm{A}$; since $\mathrm{SN} \cdots \mathrm{A} \cdots$ is prohibited, $\mathrm{Y} \neq \mathrm{S}$; and, by the NN Theorem, $\mathrm{Y} \neq \mathrm{N}$. It follows that we must have $\ell_{k+5} \neq \mathrm{N}$.

From Cases 1 and 2 we can deduce that the subsequence SSNAAZ, where $Z \in\{A, N\}$, cannot occur in the epr-sequence of a real symmetric matrix.

Case 3: $\ell_{k+5}=\mathrm{S}$. Now we have $\ell_{k} \cdots \ell_{k+5}=$ SSNAAS. By the Inheritance Theorem, $B$ has a $(k+5) \times(k+5)$ principal submatrix with an epr-sequence ending with SXNAAY, where $\mathrm{X} \in\{\mathrm{A}, \mathrm{S}, \mathrm{N}\}$ and $\mathrm{Y} \in\{\mathrm{A}, \mathrm{N}\}$. By the NN Theorem, $\mathrm{X} \neq \mathrm{N}$; and, by Proposition 2.3. $\mathrm{X} \neq \mathrm{A}$. It follows that $\mathrm{X}=\mathrm{S}$, which contradicts the assertion above.

As NAN is prohibited, we have the following corollary to Theorem 2.7 .

Corollary 2.8. The only way SNA can occur in the epr-sequence of a real symmetric matrix is in one of the two terminal sequences SNA or SNAA.

We note that the epr-sequences ANSSSNA and ANSSSNAA are attainable [3, Table 1], implying that SNA is not completely prohibited in the epr-sequence of a real symmetric matrix. Theorem 2.6 and Corollary 2.8 lead to the following observation.

Observation 2.9. Any epr-sequence of a real symmetric matrix that contains NA as a noninitial subsequence is of the form $\cdots \mathrm{NA} \overline{\mathrm{A}}$.

The following results in this section will be of particular relevance to the main results in Sections 3 and 4 .

Lemma 2.10. Let $n$ be even and $B$ be a nonsingular $n \times n$ real symmetric matrix. Then $J_{\frac{n}{2}+1}$ is not a principal submatrix of $B$. 
Proof. For the sake of contradiction, suppose, without loss of generality, that $B\left[\left\{1, \ldots, \frac{n}{2}+\right.\right.$ $1\}]=J_{\frac{n}{2}+1}$. Then the rank of the matrix consisting of the first $\frac{n}{2}+1$ columns of $B$ has rank less than $\frac{n}{2}+1$; hence, $B$ is singular, a contradiction.

Lemma 2.11. Let $n \geq 8$ be even. Let $B$ be an $n \times n$ nonsingular real symmetric matrix with every entry \pm 1 and all entries in the first row, the first column, and the diagonal equal to 1. Suppose that $\operatorname{epr}(B)=\ell_{1} \ell_{2} \cdots \ell_{n}$ and that $\ell_{4}=\mathrm{N}$. Then every row and column of $B$ has at most $\frac{n}{2}-1$ negative entries. Equivalently, every row and column of $B$ has at least $\frac{n}{2}+1$ positive entries.

Proof. Suppose $B=\left[b_{i j}\right]$ contains a row with $\frac{n}{2}$ negative entries. Let $U=\left\{3,4, \ldots, \frac{n}{2}+2\right\}$. Without loss of generality, suppose $b_{2 j}=-1$ for all $j \in U$. We claim that $B[\{1\} \cup U]=$ $J_{\frac{n}{2}+1}$. Suppose to the contrary that $B[\{1\} \cup U]$ contains a negative entry; without loss of generality, we may assume that this entry is $b_{34}$. It follows that $B[\{1,2,3,4\}]$ is nonsingular, a contradiction to $\ell_{4}=\mathrm{N}$; hence, $B[\{1\} \cup U]=J_{\frac{n}{2}+1}$. By Lemma 2.10, $B$ is singular, a contradiction to the nonsingularity of $B$. We conclude that every row and column of $B$ has at most $\frac{n}{2}-1$ negative entries.

Theorem 2.12. Let $n \geq 8$ be even and $B$ be an $n \times n$ real symmetric matrix. Suppose that $\operatorname{epr}(B)=\operatorname{ANSNSN} \cdots$. Then $B$ is singular.

Proof. Suppose to the contrary that $B$ is nonsingular. Let $B=\left[b_{i j}\right]$. By [2, Proposition 8.1], we may assume that every entry of $B$ is \pm 1 and all entries in the first row, the first column, and the diagonal are equal to 1. By Lemma 2.11, every row and column of $B$ has at least $\frac{n}{2}+1$ positive entries. Because a simultaneous permutation of the rows and columns of a matrix has no effect on its determinant, we may assume, without loss of generality, that the first $\frac{n}{2}+1$ entries in the second row (and column) are positive. Let

$$
M_{1}=\left[\begin{array}{cccc}
1 & 1 & 1 & 1 \\
1 & 1 & -1 & -1 \\
1 & -1 & 1 & -1 \\
1 & -1 & -1 & 1
\end{array}\right] \quad \text { and } \quad M_{2}=\left[\begin{array}{cccc}
1 & 1 & 1 & -1 \\
1 & 1 & -1 & 1 \\
1 & -1 & 1 & 1 \\
-1 & 1 & 1 & 1
\end{array}\right]
$$

Since $M_{1}$ and $M_{2}$ are nonsingular, they are not principal submatrices of $B$. We now show by induction on the number of negative entries in the second row that $B$ contains a row with $\frac{n}{2}$ negative entries. For the base case, first notice that the nonsingularity of $B$ implies that $B$ must have a row with at least one negative entry, as otherwise it will have a repeated row; without loss of generality, we assume that $b_{2 n}=-1$. By Lemma 2.10, $B\left[\left\{1, \ldots, \frac{n}{2}+1\right\}\right]$ has a negative entry; without loss of generality, suppose $b_{34}=-1$. Then, as $B[\{2,3,4, n\}] \neq M_{2}$, either $b_{3 n}$ or $b_{4 n}$ is negative, implying that either the third or fourth row contains two negative entries. It follows that $B$ must contain a row with two negative entries.

Now, for the inductive step, suppose the second row contains $2 \leq k \leq \frac{n}{2}-1$ negative entries. Without loss of generality, suppose $b_{2 j}=-1$ for $j \in U=\{n-k+1, \ldots, n\}$. As in the base case, Lemma 2.10 implies that $B\left[\left\{1, \ldots, \frac{n}{2}+1\right\}\right]$ has a negative entry, and, again, without loss of generality, we may assume that $b_{34}=-1$. Since $B[\{1,2, p, q\}] \neq M_{1}$ for 
$p, q \in U, b_{p q}=1$ for all $p, q \in U$. Similarly, $B[\{1,3,4, j\}] \neq M_{1}$ and $B[\{2,3,4, j\}] \neq M_{2}$ for $j \in U$, implying that $b_{3 j} \neq b_{4 j}$ for all $j \in U$; so, suppose $b_{3 j}=x_{j}$ and $b_{4 j}=-x_{j}$ for all $j \in U$. Then, as $\ell_{6}=\mathrm{N},-16\left(x_{p}-x_{q}\right)^{2}=\operatorname{det} B[\{1,2,3,4, p, q\}]=0$ for all $p, q \in U$; hence, $x_{p}=x_{q}$ for all $p, q \in U$. It follows that either the third or the fourth row contains $(n-(n-k+1)+1)+1=k+1$ negative entries. Hence, by induction, $B$ most have a row with $\frac{n}{2}$ negative entries; by Lemma 2.11, $B$ is singular, a contradiction.

We note that Theorem 2.12 cannot be generalized for $n$ odd, since, by the Inverse Theorem, AN $\overline{\operatorname{SN}}$ A is attained by $\left(A\left(C_{n}\right)\right)^{-1}$ (see [3, Observation 3.1]).

Proposition 2.13. No real symmetric matrix has an epr-sequence starting SSNSNSS ... .

Proof. Let $B=\left[b_{i j}\right]$ be a real symmetric with an epr-sequence starting SSNSNSS $\cdots$. By the Inheritance Theorem, $B$ has a $7 \times 7$ principal submatrix $B[\alpha]$ with epr-sequence $\ell_{1}^{\prime} \ell_{2}^{\prime} \mathrm{N} \ell_{4}^{\prime} \mathrm{N} \ell_{6}^{\prime} \mathrm{A}$. Without loss of generality, suppose $\alpha=\{2,3, \ldots, 8\}$. By the NN Theorem, $\ell_{2}^{\prime}, \ell_{4}^{\prime}, \ell_{6}^{\prime}$ are not N. Since NAN and NSA are prohibited, $\ell_{4}^{\prime}=S$ and $\ell_{6}^{\prime}=A$. Since ANS must be initial, $\ell_{2}^{\prime}=\mathrm{S}$. Hence, $\operatorname{epr}(B[\alpha])=\ell_{1}^{\prime}$ SNSNAA. Since ASN $\cdots \mathrm{A}$ is prohibited, $\ell_{1}^{\prime} \neq \mathrm{A}$. Then, as the epr-sequence SSNSNAA is associated with the pr-sequence 1]1101011, which is unattainable by [1, Proposition 4.1], $\ell_{1}^{\prime} \neq \mathrm{S}$; hence, $\ell_{1}^{\prime}=\mathrm{N}$, so that $\operatorname{epr}(B[\alpha])=$ NSNSNAA. We note that a simultaneous permutation of the rows and columns of a matrix has no effect on its determinant; thus, since all diagonal entries of $B[\alpha]$ are zero, and because $B$ contains a nonzero diagonal entry, we may assume, without loss of generality, that $b_{11} \neq 0$.

Let $C=B[\{1\} \cup \alpha]$ and $C=\left[c_{i j}\right]$. Then $\operatorname{epr}(C)$ starts with $\mathrm{S}$ and $\operatorname{epr}(C[\alpha])=\operatorname{epr}(B[\alpha])=$ NSNSNAA. Since every $6 \times 6$ principal submatrix of $C[\alpha]$ is nonsingular, $C[\alpha]$ contains at least two nonzero entries in each row (and column), as otherwise $C[\alpha]$ contains a $6 \times 6$ principal submatrix with a row (and column) consisting of only zeros. Moreover, we note that $c_{11}=$ $b_{11} \neq 0$; because multiplication of any row and column of a matrix by a nonzero constant preserves the rank of every submatrix, we may assume without loss of generality that $c_{11}=1$. Since $C[\alpha]$ contains a nonzero principal minor of order 2, we may assume, without loss of generality, that $\operatorname{det}((C[\alpha])[\{1,2\}]) \neq 0$; thus, $-\left(c_{23}\right)^{2}=C_{23}=(C[\alpha])[\{1,2\}] \neq 0$; hence, $c_{23} \neq 0$, and, without loss of generality, we may assume that $c_{23}=1$. Since $C[\alpha]$ contains at least two nonzero entries in each row and column, $c_{2 j} \neq 0$ for some $j \in\{4,5,6,7,8\}$; so, we may assume that $c_{24}=1$. It follows that $2 c_{34}=C_{234}=0$, and so $c_{34}=0$. Then, as $C[\alpha]$ contains at least two nonzero entries in each row and column, $c_{3 j} \neq 0$ for some $j \in\{5,6,7,8\}$; thus, suppose $c_{35}=1$. It follows that $2 c_{25}=C_{235}=0$, and so $c_{25}=0$. Now we have $-1+2 c_{12} c_{13}=C_{123}=0,-1+2 c_{12} c_{14}=C_{124}=0$ and $-1+2 c_{13} c_{15}=C_{135}=0$; it follows that $c_{12}, c_{13}, c_{14}$ and $c_{15}$ are nonzero. Let $c_{12}=x$; then $c_{13}=c_{14}=1 / 2 x$ and $c_{15}=x$. We now show that each of $c_{16}, c_{17}$ and $c_{18}$ is nonzero. Suppose to the contrary that $c_{1 j}=0$ for some $j \in\{6,7,8\}$; then $-\left(c_{i j}\right)^{2}=C_{1 i j}=0$ for all $i \in\{3,4, \ldots, 8\} \backslash\{j\}$; hence, $c_{i j}=0$ for all $i \in\{3,4, \ldots, 8\}$, implying that $C[\alpha]$ contains a row with only one nonzero entry, which is a contradiction. Without loss of generality, we may assume that $c_{16}=c_{17}=c_{18}=1$. Now, observe that $C_{145}=c_{45}\left(1-c_{45}\right)$; since all the principal minors of order 3 are zero, it follows that $c_{45}=0$ or $c_{45}=1$. Besides for the $(1,2)$-entry $x$, we have similar restrictions for all the remaining unknown entries of $C$; notice that, for $j \in\{6,7,8\}, C_{12 j}=c_{2 j}\left(2 x-c_{2 j}\right)$, $C_{13 j}=c_{3 j}\left(1 / x-c_{3 j}\right), C_{14 j}=c_{4 j}\left(1 / x-c_{4 j}\right)$ and $C_{15 j}=c_{5 j}\left(2 x-c_{5 j}\right)$. Similarly, for $k \in\{7,8\}$, $C_{16 k}=c_{6 k}\left(2-c_{6 k}\right)$. Lastly, $C_{178}=c_{78}\left(2-c_{78}\right)$. It is now clear that, besides the $(1,2)$-entry $x$, each unknown entry of $C$ is restricted to exactly two values. 
We now show that $c_{45}=1$. Suppose to the contrary that $c_{45}=0$. Since $C[\alpha]$ must contain at least two nonzero entries in each row and column, without loss of generality, we may assume that $b_{56}$ is nonzero, implying that $c_{56}=2 x$. Then $4 x c_{36}=C_{356}=0$, and therefore $c_{36}=0$. We proceed by examining the only two possibilities for the entry $c_{26}$. First, suppose $c_{26}=0$. Since all the principal minors of order 5 of $C$ are zero, $4 x c_{46}=C_{23456}=0$, implying that $c_{46}=0$. Then $C_{12456}=-4 x^{2} \neq 0$, a contradiction. So, suppose $c_{26}=2 x$. Since $4 x c_{46}=C_{246}=0, c_{46}=0$. Since $C[\alpha]$ must contain at least two nonzero entries in each row and column, suppose, without loss of generality, that $c_{47} \neq 0$; hence, $c_{47}=1 / x$. Since $2 c_{27} / x=C_{247}=0, c_{27}=0$. Now, observe that $C_{13457}=\left(-2 x+2 x^{2} c_{37}+c_{57}-x c_{37} c_{57}\right) / 2 x^{3}$ and $C_{23457}=2 c_{57} / x-2 c_{37} c_{57}$; since $C_{13457}=0$, at least one of $c_{37}$ and $c_{57}$ is nonzero; then, as $C_{23457}=0, c_{37} \neq 0$, and so $c_{37}=1 / x$. It follows that $2 c_{57} / x=C_{357}=0$, and so $c_{57}=0$. As $-4+2 c_{67}=C_{14567}=0, c_{67}=2$. Then we have $C_{234567}=0$, implying that $C[\alpha]$ has a singular $6 \times 6$ principal submatrix, which is a contradiction. We conclude that $c_{45} \neq 0$; hence, $c_{45}=1$.

Now, observe that at least one of $c_{36}, c_{37}, c_{38}, c_{46}, c_{47}$ and $c_{48}$ is nonzero, as otherwise $C[\alpha]$, which is nonsingular, would have two identical rows; thus, without loss of generality, we assume that $c_{36} \neq 0$; hence, $c_{36}=1 / x$. Similarly, at least one of $c_{27}, c_{28}, c_{57}$ and $c_{58}$ is nonzero, as otherwise $C[\{2,3,4,5,7,8\}]=(C[\alpha])[\{1,2,3,4,6,7\}]$, which is nonsingular, would have two identical rows; without loss of generality, we assume that $c_{27} \neq 0$; thus, $c_{27}=2 x$. Now the conditions $C_{236}=C_{237}=C_{247}=C_{356}=0$ imply that $c_{26}=c_{37}=c_{47}=c_{56}=0$.

Finally, we consider the only two possibilities for the entry $c_{57}$. First, suppose $c_{57}=2 x$. Then $C_{234567}=0$, a contradiction. Now, suppose $c_{57}=0$. Since $C_{234567}=-4 x^{2}\left(c_{46}-1 / x\right)^{2}$ is nonzero, $c_{46}=0$. Then $-2 c_{67}=C_{14567}=0$, and so $c_{67}=0$. Since every row and column of $C[\alpha]$ must contain at least two nonzero entries, it follows that $c_{68}$ and $c_{78}$ are nonzero, implying that $c_{68}=c_{78}=2$. The conditions $C_{278}=C_{368}=0$ imply that $c_{28}=c_{38}=0$. Hence, $C_{23678}=16 \neq 0$, a contradiction.

\section{Pr-sequences not containing three consecutive 1s}

We begin with results that forbid certain pr-sequences not containing three consecutive 1s; we then implement these in Theorem 3.10, where, for real symmetric matrices, we classify all the attainable pr-sequences not containing three consecutive $1 \mathrm{~s}$.

It is obvious from Theorem 1.1 that, with the exception of the 0th term $r_{0}^{\prime}$, we can explicitly determine each term in the pr-sequence of the inverse of a nonsingular real symmetric matrix $B$. The next result demonstrates that, when $n \geq 3, r_{0}^{\prime}$ can always be determined from $\operatorname{pr}(B)$ if this sequence does not end with 111 .

Remark 3.1. Let $n \geq 3, B$ be a nonsingular real symmetric matrix with $\left.\operatorname{pr}(B)=r_{0}\right] r_{1} \cdots r_{n-1} 1$ and $r_{0}^{\prime}$ be the 0 th term of $\operatorname{pr}\left(B^{-1}\right)$.

(i) If $r_{n-1} r_{n}=01$, then $r_{0}^{\prime}=1$.

(ii) If $r_{n-2} r_{n-1} r_{n}=011$, then $r_{0}^{\prime}=0$. 
(i) is immediate from Theorem [1.1, since $B$ obviously has a principal minor of order $n-1$ that is zero. As for (ii), first, notice that the penultimate term of $\operatorname{epr}(B)$ must be A, as NSA is prohibited; therefore, $B$ does not have a principal minor of order $n-1$ that is zero, implying that $r_{0}^{\prime}=0$.

The next proposition generalizes a particular case of [2, Lemma 4.5].

Proposition 3.2. Let $B$ be a real symmetric matrix with $\left.\operatorname{pr}(B)=r_{0}\right] r_{1} \cdots r_{n}$. Suppose that $\operatorname{pr}(B)$ does not contain three consecutive $1 s$ and that $\left.\left.r_{0}\right] r_{1} \neq 1\right] 1$. Then, for any $m$ with $1 \leq m \leq n$, there exists a principal submatrix $B^{\prime}$ of $B$ such that $\left.\operatorname{pr}\left(B^{\prime}\right)=r_{0}\right] r_{1} \cdots r_{m}$.

Proof. Let $1 \leq m \leq n$. By [2, Lemma 4.5], $B$ has a principal submatrix $B^{\prime}$ with $\operatorname{pr}\left(B^{\prime}\right)=$ $\left.r_{0}^{\prime}\right] r_{1} r_{2} \cdots r_{m}$. Since $B$ does not contain both a zero and a nonzero diagonal entry, it follows that $\left.\left.r_{0}^{\prime}\right] r_{1}=r_{0}\right] r_{1}$, and therefore $\left.\operatorname{pr}\left(B^{\prime}\right)=r_{0}\right] r_{1} \cdots r_{m}$.

Corollary 3.3. Let $\left.\sigma=r_{0}\right] r_{1} \cdots r_{n}$ be a pr-sequence not containing three consecutive $1 s$. Suppose $\left.\left.r_{0}\right] r_{1} \neq 1\right] 1$. If any initial subsequence of $\sigma$ is unattainable, then $\sigma$ is unattainable.

It was shown in [2] that appending 0 to the end of an attainable pr-sequence results in a new attainable pr-sequence; but, what if 0 is appended to an unattainable pr-sequence? For example, if we append 0 to 1]1011, which is unattainable (see [2, Table 5.4]), we obtain the attainable pr-sequence 1]10110 (see [2, Table 6.1]). However, there are some cases where appending 0 preserves unattainability. The next observation, a consequence of Corollary 3.3 , illustrates this.

Observation 3.4. Let $\left.r_{0}\right] r_{1} \cdots r_{n}$ be an unattainable pr-sequence not containing three consecutive 1 s. Suppose $\left.\left.r_{0}\right] r_{1} \neq 1\right] 1$. Then $\left.r_{0}\right] r_{1} \cdots r_{n} 0$ is also unattainable.

Propositions 3.5 and 3.7 below are corollaries to Theorem 2.12.

Proposition 3.5. Let $B$ be a real symmetric matrix with $\operatorname{epr}(B)=$ ANSNSN $\cdots$. Then, for $k \geq 1, \ell_{2 k}=\mathrm{N}$. Furthermore, $\operatorname{epr}(B)=\operatorname{ANSNSN} \overline{\operatorname{SN}} \overline{\mathrm{N}}$ or $\operatorname{epr}(B)=\operatorname{ANSNSN} \overline{\mathrm{SN}} \mathrm{A}$.

Proof. Let $k \geq 1$. By hypothesis, the first assertion holds for $k \leq 3$. Suppose $\ell_{2 k} \neq \mathrm{N}$ for some $k>3$. By the Inheritance Theorem, $B$ has a nonsingular $2 k \times 2 k$ principal submatrix with epr-sequence ANXNYN …A, where $X, Y, Z \in\{A, S, N\}$. By the NN Theorem, X and $Y$ are not N. Since NAN is prohibited, $X=Y=S$, a contradiction to Theorem 2.12. The final assertion is immediate from the NN Theorem and the fact that NAN is prohibited.

Corollary 3.6. The pr-sequence 0]10101010110 is not attainable by a real symmetric matrix.

Proof. Since 0]1010101011 satisfies the hypothesis of Observation 3.4, it suffices to show that this sequence is not attainable. Suppose that there is a real symmetric matrix $B$ with $\operatorname{pr}(B)=0] 1010101 \overline{011}$ and $\operatorname{epr}(B)=\ell_{1} \ell_{2} \cdots \ell_{n}$. Obviously, $\ell_{1}=\ell_{n}=\mathrm{A}$ and $\ell_{2}=\ell_{4}=\ell_{6}=\mathrm{N}$. Since NAN is prohibited, $\ell_{3}=\ell_{5}=\mathrm{S}$. Hence, $\operatorname{epr}(B)=$ ANSNSN $\cdots \mathrm{XA}$, where $\mathrm{X}$ is not N, which contradicts Proposition 3.5 . 
Proposition 3.7. Let $B$ be a real symmetric matrix with $\operatorname{epr}(B)=\operatorname{SNSNSN} \cdots$. Then, for $k \geq 1, \ell_{2 k}=\mathrm{N}$. Furthermore, $\operatorname{epr}(B)=\operatorname{SNSNSN} \overline{\operatorname{SN}} \overline{\mathrm{N}}$ or $\operatorname{epr}(B)=\operatorname{SNSNSNSN} A$.

Proof. Let $k \geq 1$. By hypothesis, the first assertion holds for $k \leq 3$. Suppose $\ell_{2 k} \neq N$ for some $k>3$. By the Inheritance Theorem, $B$ has a nonsingular $2 k \times 2 k$ principal submatrix with an epr-sequence XNYNZN $\cdots A$, where $X, Y, Z \in\{A, S, N\}$. By the NN Theorem, X, Y and $Z$ are not N. Since NAN is prohibited, $Y=Z=S$. Since $S N \cdots A \cdots$ is prohibited, $X \neq S$, and hence $\mathrm{X}=\mathrm{A}$, a contradiction to Theorem 2.12. As in Proposition 3.5, the final assertion follows from the NN Theorem and the fact that NAN is prohibited.

Corollary 3.8. The pr-sequence 1$] 1010101 \overline{01} 10 \overline{0}$ is not attainable by a real symmetric matrix.

Proof. Suppose there is a real symmetric matrix $B$ with $\operatorname{pr}(B)=1] 1010101 \overline{01} 10 \overline{0}$. Let $\operatorname{epr}(B)=\ell_{1} \ell_{2} \cdots \ell_{n}$. Obviously, $\ell_{1}=\mathrm{S}$ and $\ell_{2}=\ell_{4}=\ell_{6}=\mathrm{N}$. Since NAN is prohibited, $\ell_{3}=\ell_{5}=\mathrm{S}$. Hence, $\operatorname{epr}(B)=\operatorname{SNSNSN} \cdots \mathrm{XYN} \overline{\mathrm{N}}$, where $\mathrm{X}$ and $\mathrm{Y}$ are both not $\mathrm{N}$, which contradicts Proposition 3.7 .

Before proving the main result of this section, we need a lemma.

Lemma 3.9. Let $B$ be a real symmetric matrix with $\left.\operatorname{pr}(B)=r_{0}\right] r_{1} \cdots r_{n}$. Suppose $r_{1} r_{2} \cdots r_{n}$ does not contain three consecutive 1 s. Let $1 \leq k \leq \operatorname{rank}(B)-2$. If $r_{k} r_{k+1}=01$, then either $r_{k+2} r_{k+3} \cdots r_{n}=\overline{01} 1 \overline{0}$ or $r_{k+2} r_{k+3} \cdots r_{n}=\overline{01} 01 \overline{0}$

Proof. Suppose $r_{k} r_{k+1}=01$. We proceed by examining the only two possibilities for $r_{k+2}$.

Case 1: $r_{k+2}=1$. Now we have $r_{k} r_{k+1} r_{k+2}=011$. If $n=k+2$, then we are done. Now, suppose $n>k+2$. By hypothesis, $r_{k+3}=0$, and therefore, by the 0110 Theorem, $r_{k+2} r_{k+3} \cdots r_{n}=1 \overline{0}$, where $\overline{0}$ is non-empty.

Case 2: $r_{k+2}=0$. Now we have $r_{k} r_{k+1} r_{k+2}=010$. Then, as $\operatorname{rank}(B) \geq k+2$, by the 00 Theorem, $r_{k+3} \neq 0$; hence, $r_{k+3}=1$, and so $r_{k+2} r_{k+3}=01$. If $n=k+3$, then we are done. Suppose $n>k+3$. If $\operatorname{rank}(B)=k+3$, then we have $r_{k+2} r_{k+3} \cdots r_{n}=01 \overline{0}$, where $\overline{0}$ is non-empty. Suppose $\operatorname{rank}(B)>k+3$, i.e., suppose $\operatorname{rank}(B) \geq k+4$. Thus, so far we have $r_{k} r_{k+1} r_{k+2} r_{k+3}=0101$, where $r_{k+2} r_{k+3}=01$ and $1 \leq k+2 \leq \operatorname{rank}(B)-2$. Since $n$ is finite, it is evident that reimplementing the steps above by replacing $k$ with $k+2$, and repeating this process until reaching the last term of the sequence, yields the desired conclusion.

With the next theorem, we classify all the attainable pr-sequences of order $n \geq 3$ not containing three consecutive 1s.

Theorem 3.10. Let $n \geq 3$. A pr-sequence of order $n$ not containing three consecutive $1 s$ is attainable by a real symmetric matrix if and only if it is one of the following sequences.

1. 0$] 100 \overline{0}$.

2. 0$] 1 \overline{01} 01 \overline{0}$.

3. 0$] 1011 \overline{0}$.

4. 0$] 101011 \overline{0}$. 
5. 0$] 110 \overline{0}$.

6. 0$] 1101 \overline{0}$.

7. 0$] 11011 \overline{0}$.

8. 1$] 000 \overline{0}$.

9. 1]0100.

10. 1] $01 \overline{01} 01 \overline{0}$.

11. 1$] 01 \overline{01} \overline{0}$.

12. 1$] 100 \overline{0}$.

13. 1]101010̄.

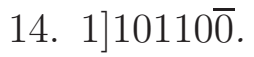

15. 1]10101100̄.

Proof. Let $B$ be a real symmetric matrix with $\left.\operatorname{pr}(B)=r_{0}\right] r_{1} \cdots r_{n}$ not containing three consecutive $1 \mathrm{~s}$. Since 0$] 0 \cdots$ is forbidden by definition, $\left.\left.\left.\left.r_{0}\right] r_{1} \in\{0] 1,1\right] 0,1\right] 1\right\}$. We proceed by examining all the possibilities for $\left.r_{0}\right] r_{1} r_{2}$.

Case $\left.\left.i: r_{0}\right] r_{1} r_{2}=0\right] 10$. If $r_{3}=0$, then, by the 00 Theorem, we have sequence (1). Suppose $r_{3}=1$. Hence, $\operatorname{pr}(B)$ starts 0$] 101 \cdots$. If $\operatorname{rank}(B)=3$, then $\left.\operatorname{pr}(B)=0\right] 101 \overline{0}$, which is sequence (2). Now, suppose $\operatorname{rank}(B)>3$. Then $r_{2} r_{3}=01$ and $1 \leq 2 \leq \operatorname{rank}(B)-2$; hence, by applying Lemma 3.9 to $\operatorname{pr}(B)$, starting with $k=2$, we have either $\operatorname{pr}(B)=0] 101 \overline{01} 01 \overline{0}$ or $\operatorname{pr}(B)=0] 101 \overline{01} 1 \overline{0}$. Hence, by Corollary [3.6, $\operatorname{pr}(B)$ is one of the sequences (2), (3) and (4).

Case ii: $\left.\left.r_{0}\right] r_{1} r_{2}=0\right] 11$. By hypothesis, $r_{3}=0$. If $\operatorname{rank}(B)=2$, then $\left.\operatorname{pr}(B)=0\right] 110 \overline{0}$, which is sequence (5). Now suppose $\operatorname{rank}(B)>2$. Then $n>3$ and, by the 00 Theorem, $r_{4} \neq 0$, implying that $r_{4}=1$. Hence, $\operatorname{pr}(B)$ starts 0$] 1101 \cdots$. If $n=4$, then we have sequence (6). Suppose $n>4$. If $r_{5}=1$, then, by the 0110 Theorem, we must have sequence (7), where $\overline{0}$ may be empty. Now, suppose $r_{5}=0$. If $n=5$, then we have sequence (6). Suppose $n>5$. Thus far we have $\operatorname{pr}(B)=0] 11010 \cdots ;$ it follows from [2, Theorem 7.2] that $r_{6}=0$, and therefore, by the 00 Theorem, we have sequence (6).

Case iii: $\left.\left.r_{0}\right] r_{1}=1\right] 0$. If $r_{2}=0$, then, by the 00 Theorem, we have sequence (8). Now, suppose $r_{2}=1$. Hence, $\operatorname{pr}(B)$ starts 1$] 01 \cdots$. If $\operatorname{rank}(B)=2$, then $\left.\operatorname{pr}(B)=1\right] 010 \overline{0}$, which is sequence (9). Now, suppose $\operatorname{rank}(B)>2$. Then $r_{1} r_{2}=01$ and $1 \leq 1 \leq \operatorname{rank}(B)-2$; hence, by applying Lemma 3.9 to $\operatorname{pr}(B)$, starting with $k=1$, we have either $\operatorname{pr}(B)=1] 01 \overline{01010}$ or $\operatorname{pr}(B)=1] 01 \overline{01} 1 \overline{0}$. Thus, $\operatorname{pr}(B)$ is either sequence (10) or (11).

Case iv: $\left.\left.r_{0}\right] r_{1}=1\right] 1$. By hypothesis, $r_{2}=0$. If $r_{3}=0$, then the 00 Theorem implies that we have sequence (12). Now, suppose $r_{3}=1$. Hence, $\operatorname{pr}(B)$ starts 1$] 101 \cdots$. Suppose $\operatorname{rank}(B)=3$; then $\operatorname{pr}(B)=1] 101 \overline{0}$, and, by [2, Theorem 4.1], $\overline{0}$ is non-empty, implying that $\operatorname{pr}(B)=1] 1010 \overline{0}$, which is sequence (13). Now, suppose $\operatorname{rank}(B)>3$. Then $r_{2} r_{3}=01$ and $1 \leq 2 \leq \operatorname{rank}(B)-2$; hence, by applying Lemma 3.9 to $\operatorname{pr}(B)$, starting with $k=2$, we have 
$\operatorname{pr}(B)=1] 101 \overline{01} 01 \overline{0}$ or $\operatorname{pr}(B)=1] 101 \overline{01} \overline{0}$; again, it follows from [2, Theorem 4.1] that in either case $\overline{0}$ must be non-empty, and therefore $\operatorname{pr}(B)=1] 101 \overline{01} 010 \overline{0}$ or $\operatorname{pr}(B)=1] 101 \overline{01} 10 \overline{0}$. Hence, by Corollary [3.8, $\operatorname{pr}(B)$ is one of the sequences (13), (14) and (15).

For the other direction, since appending 0 to the end of an attainable sequence results in another attainable sequence (see [2, Theorem 2.6]), it suffices to establish the attainability of each sequence when $\overline{0}$ is empty. We assume that the sequence under consideration has order $n \geq 3$ and provide an $n \times n$ real symmetric matrix that attains it.

1. 0$\left.] 100 \overline{0}: \operatorname{pr}\left(J_{3}\right)=0\right] 100$.

2. 0]1 $\left.\overline{01} 01 \overline{0}: \operatorname{pr}\left(\left(A\left(C_{n}\right)\right)^{-1}\right)=0\right] 1 \overline{0101}$, with $n$ odd (see [2, Lemma 3.4] and Remark 3.1).

3. 0$\left.] 1011 \overline{0}: \operatorname{pr}\left(J_{4}-2 I_{4}\right)=0\right] 1011$.

4. 0]101011̄̄: $\left.\operatorname{pr}\left(M_{0101011}\right)=0\right] 101011$, where $M_{0101011}$ appears in [2, p. 2153]

5. 0]110̄: $\left.\operatorname{pr}\left(J_{1} \oplus J_{2}\right)=0\right] 110$.

6. 0$\left.] 1101 \overline{0}: \operatorname{pr}\left(J_{4}-3 I_{4}\right)=0\right] 1101$.

7. 0$\left.] 11011 \overline{0}: \operatorname{pr}\left(J_{5}-3 I_{5}\right)=0\right] 11011$.

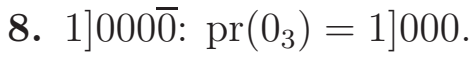

9. 1]0100̄: $\left.\operatorname{pr}\left(\left(J_{2}-I_{2}\right) \oplus 0_{1}\right)=1\right] 010$.

10. 1]0101010: $\left.\operatorname{pr}\left(A\left(P_{n}\right)\right)=1\right] 01 \overline{01} 01$, with $n$ even (see [2, Lemma 3.3]).

11. 1]01 $\left.\overline{01} 1 \overline{0}: \operatorname{pr}\left(A\left(C_{n}\right)\right)=1\right] 01 \overline{011}$, with $n$ odd (see [2, Lemma 3.4]).

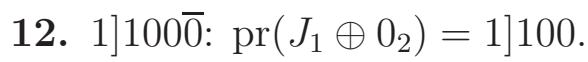

13. 1] $\left.1 \overline{01} 010 \overline{0}: \operatorname{pr}\left(\left(A\left(C_{n-1}\right)\right)^{-1} \oplus 0_{1}\right)=1\right] 1 \overline{01} 010$, with $n$ even (see [2, Lemma 3.4], Remark 3.1 and [2, Theorem 2.3]).

14. 1]10110̄: $\left.\operatorname{pr}\left(\left(J_{4}-2 I_{4}\right) \oplus 0_{1}\right)=1\right] 10110$.

15. 1]10101100̄: $\left.\operatorname{pr}\left(M_{0101011} \oplus 0_{1}\right)=1\right] 1010110$, where $M_{0101011}$ appears in [2, p. 2153].

That concludes the proof.

We conclude this section with a classification of the attainable pr-sequences that only contain three consecutive 1s in the initial subsequence 1]11. The primary motivation for including this result is its application in Section 4 .

Proposition 3.11. The epr-sequences SSNSNSTSSSNN $\bar{N}$ and SSNSTSNAA are not attainable by a real symmetric matrix. 
Proof. Suppose to the contrary that there is a real symmetric matrix $B$ with $\operatorname{epr}(B)=$

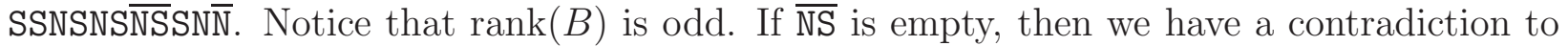
Proposition 2.13. So, suppose $\overline{\mathrm{NS}}$ is non-empty. Let $B[\alpha]$ be a nonsingular $1 \times 1$ principal submatrix of $B$. By the Schur Complement Theorem, $\operatorname{rank}(B / B[\alpha])$ is even, $\operatorname{rank}(B / B[\alpha]) \geq$ 8 , and $\operatorname{epr}(B / B[\alpha])=\mathrm{XNYNZN} \cdots$, where $\mathrm{X}, \mathrm{Y}, \mathrm{Z} \in\{\mathrm{A}, \mathrm{S}, \mathrm{N}\}$. Then, as $\operatorname{rank}(B / B[\alpha]) \geq 8$, by the NN Theorem, $\mathrm{X}, \mathrm{Y}$ and $\mathrm{Z}$ are not N. Since NAN is prohibited, $\mathrm{Y}=\mathrm{Z}=\mathrm{S}$. Thus, we have $\operatorname{epr}(B / B[\alpha])=\operatorname{XNSNSN} \cdots$, where $\mathbf{X}$ is not $\mathrm{N}$. It follows from Propositions 3.5 and 3.7 that $\operatorname{rank}(B / B[\alpha])$ is odd, a contradiction.

Now, suppose SSNSTNSNAA is attainable. Then applying [3, Observation 2.19(2)] to this sequence implies that SSNSNSTSSSN is attainable, a contradiction to the first assertion.

Corollary 3.12. The pr-sequence 1]1101010110 is not attainable by a real symmetric matrix.

Proof. Suppose that there is a real symmetric matrix $B$ with $\operatorname{pr}(B)=1] 110101 \overline{01} 1 \overline{0}$ and $\operatorname{epr}(B)=\ell_{1} \ell_{2} \cdots \ell_{n}$. Obviously, $\ell_{1}=\mathrm{S}$ and $\ell_{3}=\ell_{5}=\mathrm{N}$. By the NN Theorem, and because NAN is prohibited, $\ell_{4}=\mathrm{S}$. Since $\ell_{2}$ is not $\mathrm{N}$, it follows from Proposition 2.3 that $\ell_{2}=\mathrm{S}$. Hence, $\operatorname{epr}(B)=\operatorname{SSNSN} \cdots$. We examine two cases.

Case 1: $\overline{0}$ is empty. Notice that $\operatorname{pr}(B)=1] 110101 \overline{01} 1=1] 1101 \overline{01} 011$. Moreover, $\ell_{n}=\mathrm{A}$ and $\ell_{i}=\mathrm{N}$ for all odd $i$ with $3 \leq i \leq n-2$. Then, as NAN is prohibited, $\ell_{j}=\mathrm{S}$ for all even $j$ with $4 \leq j \leq n-3$. Therefore, we have $\operatorname{epr}(B)=$ SSNSTSNXA, where $\mathrm{X}$ is not N. Since NSA is prohibited, $\mathrm{X}=\mathrm{A}$, which contradicts Proposition 3.11 .

Case 2: $\overline{0}$ is non-empty. Thus, $\operatorname{pr}(B)=1] 110101 \overline{01} 10 \overline{0}=1] 1101 \overline{01} 0110 \overline{0}$. As in the

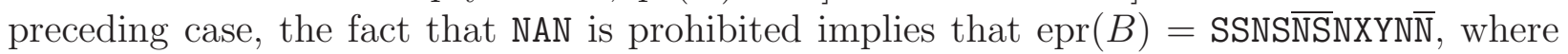
$\mathrm{X}$ and $\mathrm{Y}$ are not $\mathrm{N}$. By Theorem 2.7, $\mathrm{X}=\mathrm{S}$. Then, as NSA is prohibited, $\mathrm{Y}=\mathrm{S}$. Hence,

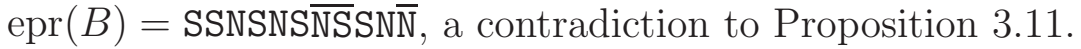

Proposition 3.13. Let $n \geq 3$. A pr-sequence $\left.r_{0}\right] r_{1} \cdots r_{n}$, with $r_{1} r_{2} \cdots r_{n}$ not containing three consecutive $1 s$, is attainable by a real symmetric matrix if and only if it is one of the sequences in Theorem 3.10 or one of the following sequences.

16. 1$] 110 \overline{0}$.

17. 1]11 $\overline{01} 01 \overline{0}$.

18. 1]11011̄̄.

Proof. Let $B$ be a real symmetric matrix with $\left.\operatorname{pr}(B)=r_{0}\right] r_{1} \cdots r_{n}$. Suppose $r_{1} r_{2} \cdots r_{n}$ does not contain three consecutive 1 s. If $\left.\left.r_{0}\right] r_{1} r_{2} \neq 1\right] 11$, then $\operatorname{pr}(B)$ does not contain three consecutive $1 \mathrm{~s}$, and therefore it is one of the sequences listed in Theorem 3.10 . Thus, suppose $\left.\left.r_{0}\right] r_{1} r_{2}=1\right] 11$. By hypothesis, $r_{3}=0$. If $n=3$, then $\operatorname{pr}(B)$ is sequence (16). So, suppose $n>3$. If $r_{4}=0$, then, by the 00 Theorem, $\operatorname{pr}(B)$ is sequence (16). Now, suppose $r_{4}=1$. Then $\operatorname{pr}(B)$ starts 1$] 1101 \cdots$. If $\operatorname{rank}(B)=4$, then $\operatorname{pr}(B)=1] 1101 \overline{0}$, which is sequence (17). Now, suppose $\operatorname{rank}(B)>4$. Hence, $r_{3} r_{4}=01$ and $1 \leq 3 \leq \operatorname{rank}(B)-2$. It follows from applying Lemma 3.9 to $\operatorname{pr}(B)$, starting with $k=3$, that $\operatorname{pr}(B)=1] 1101 \overline{0101 \overline{0}}$ or $\operatorname{pr}(B)=1] 1101 \overline{01} 1 \overline{0}$. Hence, by Corollary [3.12, $\operatorname{pr}(B)$ is either sequence (17) or sequence (18). 
For the other direction, as in Theorem 3.10, it suffices to show that each sequence is attainable when $\overline{0}$ is empty. By [2, Theorem 3.7], the sequences 1]110 and 1]11011 are attainable by $Q_{3,1}$ and $Q_{5,1}$, respectively. Finally, 1$] 11 \overline{01} 01$ is attained by $\left(A\left(F_{n}\right)\right)^{-1}$ (see [2, Lemma 3.5]), where $n$ is even and $F_{n}$ is the graph on $n$ vertices formed by adding a pendent edge to $C_{n-1}$.

\section{Epr-sequences with an $\mathrm{N}$ in every subsequence of length 3}

This section focuses on epr-sequences with an $\mathrm{N}$ in every subsequence of length 3 , and culminates with a classification of all the attainable epr-sequences with this property.

The sequence accounted for in the next result is of particular relevance to the main result at the end of this section.

Proposition 4.1. Let $n \geq 3$ and $B=\left[b_{i j}\right]$ be the $n \times n$ real symmetric matrix with $b_{i j}=$ $(i-j)^{2}$. Then $\operatorname{epr}(B)=$ NAA $\bar{N}$.

Proof. Suppose that $\operatorname{epr}(B)=\ell_{1} \ell_{2} \cdots \ell_{n}$. It is easy to verify the assertion for $n=3$. Suppose $n>3$. Obviously, $\ell_{1}=\mathrm{N}$. Let $p, q, r, s \in\{1,2, \ldots, n\}$, where $p<q<r<s$. Since every off-diagonal entry of $B$ is nonzero, we have $B_{p q}=-\left(b_{p q}\right)^{2} \neq 0$ and $B_{p q r}=2 b_{p q} b_{p r} b_{q r} \neq 0$. A simple computation reveals that the order- 4 principal minor $B_{\text {pqrs }}$ is given by

$$
\begin{gathered}
\left(b_{p s} b_{q r}\right)^{2}+\left(b_{p r} b_{q s}\right)^{2}+\left(b_{p q} b_{r s}\right)^{2}-2 b_{p r} b_{p s} b_{q r} b_{q s}-2 b_{p q} b_{p s} b_{q r} b_{r s}-2 b_{p q} b_{p r} b_{q s} b_{r s}= \\
((p-s)(q-r))^{4}+((p-r)(q-s))^{4}+((p-q)(r-s))^{4} \\
-2((p-r)(p-s)(q-r)(q-s))^{2}-2((p-q)(p-s)(q-r)(r-s))^{2} \\
-2((p-q)(p-r)(q-s)(r-s))^{2}=0 .
\end{gathered}
$$

Hence, we have $\ell_{2}=\ell_{3}=\mathrm{A}$ and $\ell_{4}=\mathrm{N}$. The conclusion now follows from Proposition 2.4.

Observation 4.2. If an attainable pr-sequence does not contain three consecutive 1s, then an attainable epr-sequence associated with it contains an $\mathrm{N}$ in every subsequence of length 3.

Remark 4.3. The converse of Observation 4.2 is false. An attainable epr-sequence starting SS $\cdots$, or starting SA $\cdots$, with an $\mathrm{N}$ in every subsequence of length 3, provides a counterexample. It can be deduced that all counterexamples are of that form, and therefore that the converse of Observation 4.2 is true if additionally we assume that the pr-sequence does not start with 1]11.

Observation 4.4. Let $n \geq 3$ and $B$ be a real symmetric matrix with $\left.\operatorname{pr}(B)=r_{0}\right] r_{1} \cdots r_{n}$. Then $\operatorname{epr}(B)$ contains an $\mathrm{N}$ in every subsequence of length 3 if and only if $r_{1} r_{2} \cdots r_{n}$ does not contain three consecutive $1 \mathrm{~s}$

Observation 4.4 suggests that we can use Theorem 3.10 and Proposition 3.13 to classify all the epr-sequences with an $\mathrm{N}$ in every subsequence of length 3 , as the pr-sequences associated with these epr-sequences must be those listed on these results. 
Theorem 4.5. Let $n \geq 3$. An epr-sequence of order $n$ with an $\mathrm{N}$ in every subsequence of length 3 is attainable by a real symmetric matrix if and only if it is one of the following sequences.

1. ANN $\bar{N}$.

2a. A $\overline{N S N A}$.

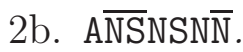

3a. ANAA.

3b. ANSSN $\bar{N}$.

4a. ANSNAA.

4b. ANSNSSN $\bar{N}$

5a. AAN $\bar{N}$.

5b. ASN $\bar{N}$.

6a. AANA

6b. ASNSNN $\bar{N}$.

7a. AANAA.

7b. ASNSSN $\bar{N}$.

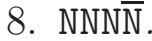

9. NSNN

10a. NST̄SNA.

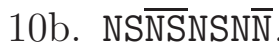

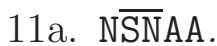

11b. N $\overline{\mathrm{SN}} \mathrm{SSN} \overline{\mathrm{N}}$.

11c. NAAN $\bar{N}$.

12. SNNT̄.

13. $\operatorname{SNSNSN\overline {N}}$.

14. $\operatorname{SNSSNN}$

15. SNSNSSNN

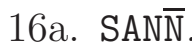


16b. SSNT̄

17a. SSTNSNA.

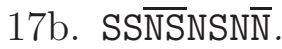

18a. SSNAA.

18b. SSNSSNN $\bar{N}$.

Proof. Let $B$ be a real symmetric matrix with $\operatorname{epr}(B)=\ell_{1} \ell_{2} \cdots \ell_{n}$. Suppose that $\operatorname{epr}(B)$ contains an $\mathrm{N}$ in every subsequence of length 3 . It follows from Observation 4.4 that $\operatorname{pr}(B)$ is one of the sequences listed in Theorem 3.10 or Proposition 3.13. We examine the 18 possible cases.

Case 1: $\operatorname{pr}(B)=0] 100 \overline{0}$. Obviously, $\operatorname{epr}(B)=\operatorname{ANN} \bar{N}$, which is sequence (1).

Case 2: $\operatorname{pr}(B)=0] 1 \overline{01} 01 \overline{0}$. First, suppose $\overline{0}$ is empty. Then, as NAN is prohibited, $\operatorname{epr}(B)=$ A $\overline{N S N}$, which is sequence $(2 \mathrm{a})$. Now, suppose $\overline{0}$ is non-empty. Similarly, since NAN is prohibited, $\operatorname{epr}(B)=A \overline{N S N S N} \bar{N}$, which is sequence $(2 \mathrm{~b})$.

Case 3: $\operatorname{pr}(B)=0] 1011 \overline{0}$. If $\overline{0}$ is empty, then, as NSA is prohibited, $\operatorname{epr}(B)=$ ANAA, which is sequence (3a). If $\overline{0}$ is non-empty, then, since NSA and NAS are prohibited, we must have ANSSN $\bar{N}$ or ANAAN $\bar{N}$; as the latter sequence is forbidden by Theorem 2.6, $\operatorname{epr}(B)$ is sequence (3b).

Case 4: $\operatorname{pr}(B)=0] 101011 \overline{0}$. Suppose $\overline{0}$ is empty. Since NAN and NSA are prohibited, $\operatorname{epr}(B)=$ ANSNAA, which is sequence (4a). Now suppose $\overline{0}$ is non-empty. Then, as NAN, NAS and NSA are prohibited, $\operatorname{epr}(B)$ is either ANSNSSN $\overline{\mathrm{N}}$ or ANSNAAN $\overline{\mathrm{N}}$; by Theorem 2.7, the latter sequence is forbidden, and thus we have sequence $(4 \mathrm{~b})$.

Case $5: \operatorname{pr}(B)=0] 110 \overline{0}$. Clearly, $\operatorname{epr}(B)=$ AAN $\bar{N}$ or $\operatorname{epr}(B)=\operatorname{ASN} \bar{N}$, which are sequences (5a) and (5b), respectively.

Case 6: $\operatorname{pr}(B)=0] 1101 \overline{0}$. If $\overline{0}$ is empty, then, as ASNA is forbidden, $\operatorname{epr}(B)=$ AANA, which is sequence $(6 \mathrm{a})$. Suppose $\overline{0}$ is non-empty. Since NAN is prohibited, and because ANS must be initial, $\operatorname{epr}(B)=\operatorname{ASNSN} \bar{N}$, which is sequence $(6 \mathrm{~b})$.

Case 7: $\operatorname{pr}(B)=0] 11011 \overline{0}$. Suppose $\overline{0}$ is empty. Since NSA and ASN $\cdots$ A are prohibited, $\operatorname{epr}(B)=$ AANAA, which is sequence (7a). Suppose $\overline{0}$ is non-empty. Moreover, suppose $\ell_{2}=\mathrm{A}$. Obviously, $\ell_{n}=\mathrm{N}$; but, as ANS must be initial, $\ell_{4}=\mathrm{A}$, and therefore Theorem 2.6 implies that $\ell_{n}=\mathrm{A}$, a contradiction. It follows that we must have $\ell_{2}=\mathrm{S}$. Since ASN $\cdots \mathrm{A} \cdots$ is prohibited, $\operatorname{epr}(B)=\operatorname{ASNSSN} \bar{N}$, which is sequence $(7 \mathrm{~b})$.

Case 8: $\operatorname{pr}(B)=1] 000 \overline{0}$. Clearly, $\operatorname{epr}(B)=$ NNN $\overline{\mathrm{N}}$, which is sequence (8).

Case 9: $\operatorname{pr}(B)=1] 010 \overline{0}$. Since NAN is prohibited, $\operatorname{epr}(B)=$ NSN $\bar{N}$, which is sequence $(9)$.

Case 10: $\operatorname{pr}(B)=1] 01 \overline{01} 01 \overline{0}$. If $\overline{0}$ is empty, then, as NAN is prohibited, $\operatorname{epr}(B)=$ NS $\overline{N S N A}$, which is sequence (10a). Similarly, if $\overline{0}$ is non-empty, $\operatorname{epr}(B)=\operatorname{NS} \overline{N S N S N} \bar{N}$, which is sequence $(10 \mathrm{~b})$.

Case 11: $\operatorname{pr}(B)=1] 01 \overline{01} 1 \overline{0}$. First, observe that $\operatorname{pr}(B)=1] 0 \overline{10} 11 \overline{0}$. Suppose $\overline{0}$ is empty. Since NSA and NAN are prohibited, $\operatorname{epr}(B)=\mathrm{N} \overline{\mathrm{SN}} \mathrm{AA}$, which is sequence (11a). Suppose $\overline{0}$ is non-empty. Moreover, suppose $\overline{10}$ is empty. Then, as NAS and NSA are prohibited, $\operatorname{epr}(B)$ is NSSNN $\bar{N}$ or NAAN $\bar{N}$, which are sequences $(11 b)$ and (11c), respectively. Finally, suppose $\overline{10}$ is

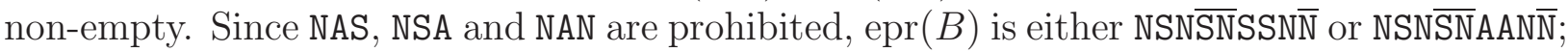


by Theorem 2.7, the latter sequence is forbidden, and therefore $\operatorname{epr}(B)$ is sequence $(11 \mathrm{~b})$, with $\overline{\mathrm{SN}}$ non-empty.

Case 12: $\operatorname{pr}(B)=1] 100 \overline{0}$. Obviously, $\operatorname{epr}(B)=\operatorname{SNN\overline {N}}$, which is sequence (12).

Case 13: $\operatorname{pr}(B)=1] 1 \overline{01} 010 \overline{0}$. Since $\mathrm{SN} \cdots \mathrm{A} \cdots$ is prohibited, it is immediate that $\operatorname{epr}(B)=\operatorname{S} \overline{N S N S N} \bar{N}$, which is sequence $(13)$.

Case 14: $\operatorname{pr}(B)=1] 10110 \overline{0}$. As in Case 13, since SN $\cdots$ A $\cdots$ is prohibited, we must have $\operatorname{epr}(B)=\operatorname{SNSSN} \bar{N}$, which is sequence $(14)$.

Case 15: $\operatorname{pr}(B)=1] 1010110 \overline{0}$. Again, as $\mathrm{SN} \cdots \mathrm{A} \cdots$ is prohibited, we must have $\operatorname{epr}(B)=$ SNSNSSN $\bar{N}$, which is sequence $(15)$.

Case 16: $\operatorname{pr}(B)=1] 110 \overline{0}$. Clearly, $\operatorname{epr}(B)$ is either $\operatorname{SANN}$ or $\operatorname{SSN} \bar{N}$, which are sequences (16a) and (16b), respectively.

Case 17: $\operatorname{pr}(B)=1] 11 \overline{01} 01 \overline{0}$. Since SAN $\cdots A \cdots$ and SAN $\cdots \mathrm{S} \cdots$ are prohibited by Proposition 2.3. $\ell_{2}=\mathrm{S}$. Suppose $\overline{0}$ is empty. Then, as NAN is prohibited, epr $(B)=\operatorname{SS} \overline{N S N A}$, which is sequence (17a). Suppose $\overline{0}$ is non-empty. Similarly, since NAN is prohibited, $\operatorname{epr}(B)=$

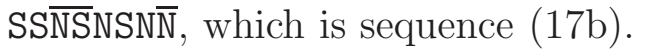

Case 18: $\operatorname{pr}(B)=1] 11011 \overline{0}$. As in the preceding case, we must have $\ell_{2}=\mathrm{S}$. Suppose $\overline{0}$ is empty. Since NSA is prohibited, $\operatorname{epr}(B)=$ SSNAA, which is sequence (18a). Suppose $\overline{0}$ is non-empty. Hence, the fact that NAS and NSA are prohibited implies that $\operatorname{epr}(B)$ is either

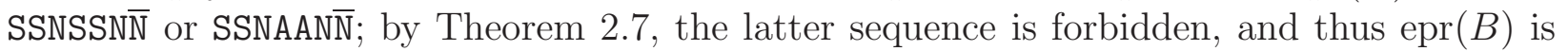
sequence $(18 \mathrm{~b})$.

For the other direction, we show that all the sequences listed are attainable, and assume that the sequence under consideration has order $n \geq 3$. Sequence (1) is attained by $J_{n}$. Sequence (2a) is attained by $A\left(\left(C_{n}\right)^{-1}\right)$ (see [3. Observation 3.1] and the Inverse Theorem), when $\overline{\mathrm{NS}}$ is non-empty, and by [3, Proposition 2.17], when $\overline{\mathrm{NS}}$ is empty. As for (2b), applying [3. Observation 2.19(1)] to (2a), results in this sequence. Sequence (3a) is attainable by [3, Proposition 2.17]. Sequence (3b) is attainable by applying [3, Observation 2.19(1)] to (3a). Sequence (4a) is attainable by [3, Table 1], and (4b) results from applying [3. Observation $2.19(1)$ ] to (4a). Sequences (5a) and (5b) are attainable by [3, Theorem 4.6]. Sequence (6a) is attainable by [3, Proposition 2.17], and (6b) results from applying [3, Observation $2.19(1)$ ] to (6a). Sequence (7a) is attainable by [3, Proposition 2.17], and (7b) results from applying [3, Observation 2.19(1)] to (7a). Sequence (8) is attained by $0_{n}$. As for (9), applying [3, Observation 2.19(1)] to the sequence NA, which is attained by $J_{2}-I_{2}$, results in this sequence. Sequence (10a) is attainable by [3, Observation 3.1], and (10b) results from applying [3, Observation 2.19(1)] to (10a). Sequence (11a) is attainable by [3, Observation 3.1], while (11b) is obtained from applying [3. Observation 2.19(1)] to (11a). Sequence (11c) is attainable by Proposition 4.1. Sequence (12) is attainable by [3, Theorem 4.6]. Sequences (13), (14) and (15) result from applying [3, Observation 2.19(2)] to (2a), (3a) and (4a), respectively. Sequences (16a) and (16b) are attainable by [3, Theorem 4.6]. According to Proposition 3.13 , the sequence 1]11 $\overline{01} 01$ is attainable; by Proposition 2.3 , and because NAN is prohibited, an attainable epr-sequence associated with this pr-sequence, must be SSTSNA, which is sequence (17a). Sequence (17b) results from applying [3, Observation 2.19(2)] to (17a). Sequence (18a) is attainable by [3, Table 5], and (18b) is attainable by [3, Corollary $2.20(2)]$.

If an epr-sequence is attainable, then the pr-sequence associated with it must be attain- 
able. The converse is not true; this is because an epr-sequence associated with a pr-sequence may not be unique, since a 1 in the pr-sequence can correspond to an A or $\mathrm{S}$ in the eprsequence. For example, the epr-sequences NSSN and NAAN, which are associated with the pr-sequence 1]0110, are each attainable by a real symmetric matrix (see [3, Table 4]). We now show that, for real symmetric matrices, almost all attainable pr-sequences not containing three consecutive 1s are associated with a unique epr-sequence.

Proposition 4.6. Let $n \geq 3$ and $\sigma$ be a pr-sequence that is attainable by an $n \times n$ real symmetric matrix. Suppose $\sigma$ does not contain three consecutive $1 s, \sigma \neq 0] 110 \overline{0}$ and that $\sigma \neq 1] 0110 \overline{0}$. Then there is a unique attainable epr-sequence associated with $\sigma$.

Proof. Since the attainable epr-sequences associated with pr-sequences not containing three consecutive 1s are the epr-sequences (1a)-(15) listed in Theorem 4.5, an attainable eprsequence associated with $\sigma$ must be one of these sequences. Note that $\sigma$ is not associated with any of the epr-sequences (16a)-(18b), as these are the epr-sequences that are associated with the pr-sequences listed in Proposition 3.13. We consider two cases.

Case 1: $\sigma=1] 010 \overline{10} 110 \overline{0}$. Observe that $\sigma$ is associated with the epr-sequence $(11 \mathrm{~b})$ in Theorem 4.5, with $\overline{\mathrm{SN}}$ non-empty. It is easy to see that $\sigma$ is not associated with any of the other epr-sequences listed in Theorem 4.5, thereby establishing the uniqueness of the associated epr-sequence (11b).

Case 2: $\sigma \neq 1] 010 \overline{10} 110 \overline{0}$. Then, as $\sigma \neq 1] 0110 \overline{0}$, the epr-sequences (11b) and (11c) in Theorem 4.5 are not associated with $\sigma$. Also, it is clear that $\sigma$ is not associated with the epr-sequence (11a) in Theorem 4.5. Since $\sigma \neq 0] 110 \overline{0}$, the epr-sequences (5a) and (5b) in Theorem 4.5 are not associated with $\sigma$. Thus far we have that $\sigma$ is not associated with any of the epr-sequences (5a), (5b), (11a), (11b) or (11c). Hence, $\sigma$ must be one of the pr-sequences (1)-(4), (6)-(10) or (12)-(15) in Theorem 3.10. Now, by considering all the possible cases, one easily verifies that an attainable epr-sequence associated with $\sigma$, which must be listed in Theorem 4.5, is unique.

\section{Acknowledgements}

The author expresses his gratitude to Dr. Leslie Hogben, for introducing him to this problem, and for her guidance.

\section{References}

[1] W. Barrett, S. Butler, M. Catral, S. M. Fallat, H.T. Hall, L. Hogben, P. van den Driessche, M. Young. The principal rank characteristic sequence over various fields. Linear Algebra Appl. 2014;459: 222-236.

[2] R. A. Brualdi, L. Deaett, D. D. Olesky, P. van den Driessche. The principal rank characteristic sequence of a real symmetric matrix. Linear Algebra Appl. 2012;436: $2137-2155$. 
[3] S. Butler, M. Catral, S. M. Fallat, H. T. Hall, L. Hogben, P. van den Driessche, M. Young. The enhanced principal rank characteristic sequence. Linear Algebra Appl. 2016;498: 181-200.

[4] S. M. Fallat, D. D. Olesky, P. van den Driessche. The enhanced principal rank characteristic sequence for skew-symmetric matrices. Linear Algebra Appl. 2016;498: 366-377.

[5] O. Holtz, H. Schneider. Open problems on GKK $\tau$-matrices. Linear Algebra Appl. 2002;345: 263-267. 